Original Article

\title{
PHYTOCHEMICAL PROFILING OF MEDICALLY SIGNIFICANT CRUDE EXTRACT USING GC-MS ANALYSIS
}

\author{
MARUTHAMUTHU RAJADURAI ${ }^{1}$, V. MAITHILI' ${ }^{2}$, R. ARUNAZHAGI ${ }^{3}$, V. YOGESH ${ }^{4}$ \\ PG and Research Department of Biotechnology and Bioinformatics, Bishop Heber College, Tiruchirappalli 620001, India \\ Email: mr.rajadurai@gmail.com
}

Received: 10 Jul 2018, Revised and Accepted: 07 Sep 2018

\begin{abstract}
Objective: The objective of this research is to identify the phytochemical constitutions present in Natural crude extract which obtained from Thumlappati district.

Methods: Kidney stone is one of the most clinical disorder arising nowadays. They are existing due to the depletion of the urine and disproportionate execration of the components such as oxalate, phosphate, uric, cysteine, and struvite. Many alopathy medicine are not effectively curable in the case of kidney stone, consequently people are in need of traditional medicine system. Thus there is a great demand for research on potential inhibitor from natural products for dissolving kidney stone. In present work deals with an unknown crude extract collected from G. Thumlappati, Battalagundu Dindugal district Tamil Nadu. The crude extract of phytochemical are analyzed by using GCMS method.
\end{abstract}

Results: Thus the sample has some bioactive compound to discharge the stone particles. So we subjected the crude extract sample to GC-MS process which reveals 210 compounds in 21 different peaks.

Conclusion: This studies forms a basis for the biological characterization and importance of bioactive compounds were identified.

Keywords: Kidney stone, Crude extract, GCMS analysis, Bioactive compounds

(C) 2018 The Authors. Published by Innovare Academic Sciences Pvt Ltd. This is an open access article under the CC BY license (http://creativecommons.org/licenses/by/4.0/) DOI: http://dx.doi.org/10.22159/ijcpr.2018v10i6.30966

\section{INTRODUCTION}

History reveals that, every civilization in the world used plants as their derivatives for treatment (or) prevention of diseases. Plants had been used as traditional health care system from the centuries and is a major source of the therapeutic agents for curing the human diseases. In the last few years more than 13000 plants have been studied for the various diseases among these some medicinal properties of plants have been documented by researchers [1-3]. In India the Traditional medicinal system using medicinal plants are Ayurveda, siddha, homeopathy, etc., to treat various diseases [4]. Traditional plant based medicines for primary health care need is followed in underdeveloped countries of about $80 \%$ of world's population (WHO) [5]. A large portion of the world population, especially in developing countries depends on the traditional system of medicine for a variety of disease. Traditional medicine have become more popular in the treatment of many diseases due to popular belief that green medicine is safe, and with less side effects. Traditional medicine is the sum of knowledge skills and practices based on the theories, beliefs, and experience indigenous to different culture that are used to maintain health and also to prevent, diagnose, improve or treat physical and mental illness [6]. It is also believed that crude extract from medicinal plants are biologically active than isolated compounds due to their synergistic effects [7]. Therefore Kidney stones are hard deposits made of minerals and salts on the inner lining of the kidney. The world Health Organization reported 35 million peoples are affected by kidney stone [8]. In India states like Andhra Pradesh, Odisha and Tamil Nadu were worst affected by Kidney stone. The scientific drugs are taken by the affected people are not much benefited only during the time of drugs consumption there are reveling the problem and pain. After few month again they were affected by the stone formation due to their food habits and the environment. So we have a small step to completely cure the stone from the urinary tract and not allowing to form the stone again by our unknown crude sample.

In this study the Gas Chromatography Mass Spectroscopy (GC-MS) method was carried out in the methanol of crude extract for the phytochemical analysis followed by qualitative and quantitative determination of the compounds. This crude possess various medicinal properties, the aim of this study was to identify the phytocompounds in the methanol of crude extract and to identify each specific compound with their concentration by GC-MS analysis. Extraction of several active phytocompounds from these extract leadsto some high activity profile drugs.

\section{MATERIALS AND METHODS}

\section{Collection of extract}

On every Sunday early morning, the $60 \mathrm{y}$ old folker persons (men and women) providing the mixed plant crude extract to the people affected from kidney stones as a liquid medicine at free of cost, They are so delight to do as a service to the public. By hearing the statement we collected sample G. Thumlappati, Battalagundu Dindugal district Tamil Nadu, India, through interview and questionaries' from folker peoples, collected the crude sample for further research.

\section{Preparation of extract}

$500 \mathrm{ml}$ of crude extract were heated at the temperature not exceeding the boiling point. The fine paste were obtained. Required quantity of the sample was weighted, transferred to the conical flask, and diluted with methanol in the ratio of 1:2 until the paste was fully immersed, the flask was shaken every hour for the first $6 \mathrm{~h}$ and incubated overnight, then filtered through what man No.1 filter paper. The methanol sample may be contains polar and non-polar components of the material and $4 \mu \mathrm{l}$ of methanol sample was employed in GCMS analysis.

\section{GCMS analysis}

The GC-MS analysis was carried out using a Clarus 500 PerkinElmer (Auto system XL) Gas Chromatograph equipped and coupled to a mass detector Turbo mass gold-Perkin Elmer Turbo mass 5.1 spectrometer with an Elite-1 (100\% Dimethyl poly siloxane), 30m x $0.25 \mathrm{~mm}$ ID x $1 \mu \mathrm{m}$ of capillary column. The instrument was set to an initial temperature of $110 \mathrm{oC}$, and maintained at this temperature for $2 \mathrm{~min}$. At the end of this period the oven temperature was rose up to $280^{\circ} \mathrm{C}$, at the rate of an increase of $5 \mathrm{oC} / \mathrm{min}$, and maintained for 9 
min. Injection port temperature was ensured as $250 \mathrm{oC}$ and Helium flow rate as one $\mathrm{ml} / \mathrm{min}$. The ionization voltage was $70 \mathrm{eV}$. The samples were injected in split mode as 10:1. Mass spectral scan range was set at $45-450(\mathrm{~m} / \mathrm{z})$

\section{Identification of phytocompounds}

Interpretation on Mass-Spectrum GC-MS was conducted using the database of National institute Standard and Technology (NIST) having more 62,000 patterns. The spectrum of the unknown components was compared with the spectrum of known components stored in the NIST library. The name, molecular weight and retention time of the components of the test materials were ascertained.

\section{RESULTS AND DISCUSSION}

At present the crude sample was utilized by tribal people residing at different corners of the district and also by rural and urban persons. We observed that the region of G. Thumalappati has lot of traditional utility of medicinal plants and herbs for diseases. But the folker were not willing to reveal the compounds of the crude sample. GCMS is one of the technique to identify the bioactive constituents of long chain branched chain hydrocarbons, acids, alcohols, esters etc. GCMS analysis was done using the organic solvent methanol it shows the presence of different 210 compounds in the crude sample. The spectrum profile of GCMS confirmed the 21 major peaks with the retention time $10.257,10.781,12.326,13.138,14.657,15.222,16.828$, $21.892,23.428,24.654,25.966,28.821,31.006,32.161,33.480,36.490,3$ $7.941,38.799,40.163,41.134,42.157$. The studies on the active principles in the plant crude sample of methanolic extract by GCMS analysis clearly showed the presence of 210 compounds with their retention time (RT), molecular weight (MW) are presented in table 1 . The GCMS chromatogram of the 21 peak of the compounds detected was shown in fig. 1. The highest peak area \% (15) is 29.742 and the lowest peak area \% (1) is 0.010 . By comparing the GCMS compound against with traditional plants using Dr. Dukes photochemical and ethanobotanical database, almost maximum number of crude sample compounds are identify insarcostemma acidum, Hymenocardia acida, Cicca acida, Rumex aceosella, Phyllanthus acidus, Citrus auratum, Citrus acida, Uncaria acida, Citrus sinesis, Elephantopus scaber, Tribulus cistoidesplants which has a property of Inhibition formation of uric acid.

Table 1: Compound detected in the methanol extract of crude sample

\begin{tabular}{|c|c|c|c|}
\hline S. No. & Compound name & Retenton time (min) & Molecular weight \\
\hline 1 & Benzoic acid & 10.257 & 122 \\
\hline 2 & Benzoic acid, silver(1+) salt & 10.257 & 228 \\
\hline 3 & Heptanediamide, N,N'-di-benzoyloxy & 10.257 & 398 \\
\hline 4 & Benzoic acid & 10.257 & 122 \\
\hline 5 & Cyclobutane-1,1-dicarboxamide, N,N'-di-benzoyloxy- & 10.257 & 382 \\
\hline 6 & 2,4-Dinitrophennylhydrazone of ribose tetrabenzoate & 10.257 & 746 \\
\hline 7 & Methanol, oxo-, benzoate & 10.257 & 150 \\
\hline 8 & 4-Piperidinepropanoic acid, 1-benzoyl-3-(2-chloroethyl)-, ethyl & 10.257 & 351 \\
\hline 9 & Cyclopropanecarboxamide, N-benzoyloxy- & 10.257 & 205 \\
\hline 10 & 1-0-Monoacetyl-2,3-0-dibenzoyl-d-ribofuranose & 10.257 & 400 \\
\hline 11 & Phenol, 4-ethenyl-, acetate & 10.781 & 162 \\
\hline 12 & Benzofuran, 2,3-dihydro- & 10.781 & 120 \\
\hline 13 & 4-Ethoxystyrene & 10.781 & 148 \\
\hline 14 & Benzaldehyde, 4-methyl- & 10.781 & 120 \\
\hline 15 & Benzene, (ethenyloxy)- & 10.781 & 120 \\
\hline 16 & Benzaldehyde, 3-methyl- & 10.781 & 120 \\
\hline 17 & 4-tert-Butoxystyrene & 10.781 & 176 \\
\hline 18 & Benzaldehyde, 2-methyl- & 10.781 & 120 \\
\hline 19 & 6-Methylenebicyclo[3.2.0]hept-3-en-2-one & 10.781 & 120 \\
\hline 20 & Bicyclo[4.2.0]octa-1,3,5-trien-7-ol & 10.781 & 120 \\
\hline 21 & dl-Mevalonic acid lactone & 12.326 & 130 \\
\hline 22 & 2-Hexene, 1-methoxy-, (E)- & 12.326 & 114 \\
\hline 23 & Oxirane, butyl- & 12.326 & 100 \\
\hline 24 & (2,3-Dimethyloxiranyl)methanol & 12.326 & 102 \\
\hline 25 & trans-3-Penten-2-ol & 12.326 & 86 \\
\hline 26 & 2(3H)-Furanone, dihydro-3-hydroxy-4,4-dimethyl-, (.+/-.)- & 12.326 & 130 \\
\hline 27 & 2-Nonanone & 12.326 & 142 \\
\hline 28 & Pentane, 1-(2-propenyloxy)- & 12.326 & 128 \\
\hline 29 & Cyclooctyl S-2-(dimethylamino)ethyl propylphosphonofluoridate & 12.326 & 321 \\
\hline 30 & 2,6-Octadiene-4,5-diol & 12.326 & 142 \\
\hline 31 & 2-Methoxy-4-vinylphenol & 13.138 & 150 \\
\hline 32 & 4-Hydroxy-2-methylacetophenone & 13.138 & 150 \\
\hline 33 & Ethanone, 1-(2-hydroxy-5-methylphenyl)- & 13.138 & 150 \\
\hline 34 & 4-Hydroxy-3-methylacetophenone & 13.138 & 150 \\
\hline 35 & 3-Methoxyacetophenone & 13.138 & 150 \\
\hline 36 & Benzene, 1-ethoxy-4-ethyl- & 13.138 & 150 \\
\hline 37 & Ethanone, 1-[5-(1-hydroxyethylidene)-1,3-cyclopentadien-1-yl]- & 13.138 & 150 \\
\hline 38 & Phenol, m-tert-butyl- & 13.138 & 150 \\
\hline 39 & Phenol, 2-(1,1-dimethylethyl)- & 13.138 & 150 \\
\hline 40 & 1-(4-Hydroxymethylphenyl)ethanone & 13.138 & 150 \\
\hline 41 & $1(3 \mathrm{H})$-Isobenzofuranone & 14.657 & 134 \\
\hline 42 & Benzoic acid, 2-(hydroxymethyl)- & 14.657 & 152 \\
\hline 43 & Benzoyl bromide & 14.657 & 184 \\
\hline 44 & Ethanone, 2,2-dibromo-1-phenyl- & 14.657 & 276 \\
\hline 45 & Ethanone, 2,2-dihydroxy-1-phenyl- & 14.657 & 152 \\
\hline 46 & beta.-Benzilmonoxime & 14.657 & 225 \\
\hline 47 & Benzhydrazide, N2-(2-methoxy-5-nitrobenzylideno)- & 14.657 & 299 \\
\hline 48 & N,N'-(4,5-Dimethyl-1,3-phenylene) bisbenzamide & 14.657 & 344 \\
\hline
\end{tabular}




\begin{tabular}{|c|c|c|c|}
\hline 49 & Benzoic acid, 3,5-difluophenyl ester & 14.657 & 234 \\
\hline 50 & . alpha.,. alpha.-Dichloroacetophenone & 14.657 & 188 \\
\hline 51 & Dodecane, 1-chloro- & 15.222 & 204 \\
\hline 52 & 1-Chloroundecane & 15.222 & 190 \\
\hline 53 & Decane, 1-chloro- & 15.222 & 176 \\
\hline 54 & Tetradecane, 1-chloro- & 15.222 & 232 \\
\hline 55 & Nonane, 1-chloro- & 15.222 & 162 \\
\hline 56 & Hexadecane, 1,16-dichloro- & 15.222 & 294 \\
\hline 57 & n-Dodecylpyridinium chloride & 15.222 & 283 \\
\hline 58 & Hexadecane, 1-chloro- & 15.222 & 260 \\
\hline 59 & Octane, 1-chloro- & 15.222 & 148 \\
\hline 60 & 1-Octadecanesulphonyl chloride & 15.222 & 352 \\
\hline 61 & 1-Undecanol & 16.828 & 172 \\
\hline 62 & Cyclodecane, methyl- & 16.828 & 154 \\
\hline 63 & Cyclopropane, nonyl- & 16.828 & 168 \\
\hline 64 & E-11,13-Tetradecadien-1-ol & 16.828 & 210 \\
\hline 65 & Cyclodecane & 16.828 & 140 \\
\hline 66 & 1-Decanol & 16.828 & 158 \\
\hline 67 & 3-Tetradecene, (Z)- & 16.828 & 196 \\
\hline 68 & Cyclooctane, 1,2-dimethyl- & 16.828 & 140 \\
\hline 69 & 3-Dodecene, (E)- & 16.828 & 168 \\
\hline 70 & Cyclooctane, methyl- & 16.828 & 126 \\
\hline 71 & 3-tert-Butyl-4-hydroxyanisole & 21.892 & 180 \\
\hline 72 & Ethanone, 1-(3,4-dimethoxyphenyl)- & 21.892 & 180 \\
\hline 73 & 3',5'-Dimethoxyacetophenone & 21.892 & 180 \\
\hline 74 & 4-((1E)-3-Hydroxy-1-propenyl)-2-methoxyphenol & 21.892 & 180 \\
\hline 75 & Phenol, 3-(1,1-dimethylethyl)-4-methoxy- & 21.892 & 180 \\
\hline 76 & (+)-s-2-Phenethanamine, 1-methyl-N-vanillyl- & 21.892 & 271 \\
\hline 77 & Ethanone, 1-(2,5-dimethoxyphenyl)- & 21.892 & 180 \\
\hline 78 & (+-)-2-Phenethanamine, 1-methyl-N-vanillyl- & 21.892 & 271 \\
\hline 79 & 2,5-Dimethoxy-4-ethylamphetamine & 21.892 & 223 \\
\hline 80 & 1,2,4-Cyclopentanetrione, 3-(2-pentenyl)- & 21.892 & 180 \\
\hline 81 & Tetradecane, 1 -chloro- & 23.428 & 232 \\
\hline 82 & Dodecane, 1-chloro- & 23.428 & 204 \\
\hline 83 & Hexadecane, 1-chloro- & 23.428 & 260 \\
\hline 84 & 1-Chloroundecane & 23.428 & 190 \\
\hline 85 & Decane, 1-chloro- & 23.428 & 176 \\
\hline 86 & Hexadecane, 1,16-dichloro & 23.428 & 294 \\
\hline 87 & 1-Octadecanesulphonyl chloride & 23.428 & 352 \\
\hline 88 & Nonadecane, 1-chloro- & 23.428 & 302 \\
\hline 89 & Octadecane, 1-chloro- & 23.428 & 288 \\
\hline 90 & Nonane, 1-chloro- & 23.428 & 162 \\
\hline 91 & 1-Hexadecanol & 24.654 & 242 \\
\hline 92 & n-Tridecan-1-ol & 24.654 & 200 \\
\hline 93 & Cyclotetradecane & 24.654 & 196 \\
\hline 94 & Hexadecen-1-ol, trans-9- & 24.654 & 240 \\
\hline 95 & 3-Hexadecene, (Z)- & 24.654 & 224 \\
\hline 96 & 5-Octadecene, (E)- & 24.654 & 252 \\
\hline 97 & 7-Hexadecene, (Z)- & 24.654 & 224 \\
\hline 98 & n-Heptadecanol-1 & 24.654 & 256 \\
\hline 99 & 1-Undecanol & 24.654 & 172 \\
\hline 100 & Cetene & 24.654 & 224 \\
\hline 101 & 2-Propenoic acid, 3-(4-hydroxy-3-methoxyphenyl)-, methyl ester & 25.966 & 208 \\
\hline 102 & 2-Propenoic acid, 3-[4-(acetyloxy)-3-methoxyphenyl]-, methyl es & 25.966 & 208 \\
\hline 103 & 1,2-Dimethoxy-4-(3-methoxy-1-propenyl)benzene & 25.966 & 208 \\
\hline 104 & 2-Propenoic acid, 3-(2,4-dimethoxyphenyl)-, (E)- & 25.966 & 208 \\
\hline 105 & 3,5-Dimethoxycinnamic acid & 25.966 & 208 \\
\hline 106 & 2,3-Dimethoxycinnamic acid & 25.966 & 208 \\
\hline 107 & 1H-1,3-Benzimidazole-6-carboxylic acid, 2-mercapto-, methyl est & 25.966 & 208 \\
\hline 108 & 2,5-Dimethoxycinnamic acid & 25.966 & 208 \\
\hline 109 & 4-Methyl-3,5-dinitrobenzamide & 25.966 & 225 \\
\hline 110 & 3,5-Dimethoxy-4-hydroxycinnamaldehyde & 25.966 & 208 \\
\hline 111 & 1-Octanol, 2-butyl- & 28.821 & 186 \\
\hline 112 & 2-Ethyl-1-dodecanol & 28.821 & 214 \\
\hline 113 & 2-Dodecanol & 28.821 & 186 \\
\hline 114 & Methoxyacetic acid, pentadecyl ester & 28.821 & 300 \\
\hline 115 & 2-Methyl-1-undecanol & 28.821 & 186 \\
\hline 116 & 1-Dodecanol, 2-methyl-, (S)- & 28.821 & 200 \\
\hline 117 & Isobutyl tetradecyl carbonate & 28.821 & 314 \\
\hline 118 & 1-Hexadecanol, 2-methyl- & 28.821 & 256 \\
\hline 119 & 2-Hexyl-1-octanol & 28.821 & 214 \\
\hline 120 & 2-Hexadecanol & 28.821 & 242 \\
\hline 121 & 2-Propenoic acid, 3-(4-hydroxy-3-methoxyphenyl)-, methyl ester & 31.006 & 208 \\
\hline 122 & 2-Propenoic acid, 3-[4-(acetyloxy)-3-methoxyphenyl]-, methyl es & 31.006 & 250 \\
\hline
\end{tabular}




\begin{tabular}{|c|c|c|c|}
\hline 123 & 1,2-Dimethoxy-4-(3-methoxy-1-propenyl)benzene & 31.006 & 208 \\
\hline 124 & 1H-1,3-Benzimidazole-6-carboxylic acid, 2-mercapto-, methyl est & 31.006 & 208 \\
\hline 125 & 2-Propenoic acid, 3-(2,4-dimethoxyphenyl)-, (E)- & 31.006 & 208 \\
\hline 126 & 2,5-Dimethoxycinnamic acid & 31.006 & 208 \\
\hline 127 & $\begin{array}{l}\text { 2-Propenoic acid, 3-(2,3-dimethoxyphenyl)-, (E)- } \\
8166872\end{array}$ & 31.006 & 208 \\
\hline 128 & trans-2,5-Dimethoxycinnamic acid & 31.006 & 208 \\
\hline 129 & 3,5-Dimethoxycinnamic acid & 31.006 & 208 \\
\hline 130 & 1,3-Benzenedicarboxylic acid, 4-methyl-, dimethyl ester & 31.006 & 208 \\
\hline 131 & 6,9,12,15-Docosatetraenoic acid, methyl ester & 32.161 & 346 \\
\hline 132 & Cyclopropanepentanoic acid, 2-undecyl-, methyl ester, trans- & 32.161 & 310 \\
\hline 133 & Oxiraneundecanoic acid, 3-pentyl-, methyl ester, cis- & 32.161 & 312 \\
\hline 134 & Cyclopropanedodecanoic acid, 2-octyl-, methyl ester & 32.161 & 366 \\
\hline 135 & Oxiraneundecanoic acid, 3-pentyl-, methyl ester, trans- & 32.161 & 312 \\
\hline 136 & Methyl 11-hexadecenoate & 32.161 & 268 \\
\hline 137 & Butyl 6,9,12-hexadecatrienoate & 32.161 & 306 \\
\hline 138 & Octadecanoic acid, 9,10-dichloro-, methyl ester & 32.161 & 366 \\
\hline 139 & 14-Methylpentadec-9-enoic acid methyl ester & 32.161 & 268 \\
\hline 140 & Methyl 9-eicosenoate & 32.161 & 324 \\
\hline 141 & Acetic acid, 2-diacetylamino-1-methyl-1-propenyl ester & 33.480 & 213 \\
\hline 142 & 6,6-Dimethyl-1,4-dioxa-spiro[4.5]dec-7-ene & 33.480 & 168 \\
\hline 143 & 1-Nitro-. beta.-d-arabinofuranose, tetraacetate & 33.480 & 363 \\
\hline 144 & 1-Nitro-2-acetamido-1,2-dideoxy-d-glucitol & 33.480 & 252 \\
\hline 145 & N,N-Diethyl-N'-(1-naphthyl)ethylenediamine & 33.480 & 242 \\
\hline 146 & DL-Leucine, N-DL-leucyl- & 33.480 & 244 \\
\hline 147 & 1,16-Cyclocorynan-17-oic acid, 19,20-didehydro-, methyl ester, & 33.480 & 322 \\
\hline 148 & 1-Nitro-2-acetamido-1,2-dideoxy-d-mannitol & 33.480 & 352 \\
\hline 149 & 9-Oxabicyclo[3.3.1]nonane-2,6-dione, 2-oxime-6-ethylene ketal & 33.480 & 213 \\
\hline 150 & Malonodihydrazide, 2-(3-butoxy-2-hydroxypropyl)- & 33.480 & 262 \\
\hline 151 & trans-13-Octadecenoic acid, methyl ester & 36.490 & 296 \\
\hline 152 & 11-Octadecenoic acid, methyl ester & 36.490 & 296 \\
\hline 153 & 6-Octadecenoic acid, methyl ester, (Z)- & 36.490 & 296 \\
\hline 154 & 10-Octadecenoic acid, methyl ester & 36.490 & 296 \\
\hline 155 & 6-Octadecenoic acid, methyl ester & 36.490 & 296 \\
\hline 156 & cis-13-Octadecenoic acid, methyl ester & 36.490 & 296 \\
\hline 157 & 13-Octadecenoic acid, methyl ester & 36.490 & 296 \\
\hline 158 & 16-Octadecenoic acid, methyl ester & 36.490 & 296 \\
\hline 159 & 9-Octadecenoic acid (Z)-, methyl ester & 36.490 & 296 \\
\hline 160 & 9-Octadecenoic acid (Z)-, methyl ester & 36.490 & 296 \\
\hline 161 & trans-13-Octadecenoic acid, methyl ester & 37.941 & 296 \\
\hline 162 & 11-Octadecenoic acid, methyl ester & 37.941 & 296 \\
\hline 163 & 10-Octadecenoic acid, methyl ester & 37.941 & 296 \\
\hline 164 & cis-13-Octadecenoic acid, methyl ester & 37.941 & 296 \\
\hline 165 & 13-Octadecenoic acid, methyl ester & 37.941 & 296 \\
\hline 166 & 16-Octadecenoic acid, methyl ester & 37.941 & 296 \\
\hline 167 & 6-Octadecenoic acid, methyl ester & 37.941 & 296 \\
\hline 168 & 14-Octadecenoic acid, methyl ester & 37.941 & 296 \\
\hline 169 & 6-Octadecenoic acid, methyl ester, (Z)- & 37.941 & 296 \\
\hline 170 & 9-Octadecenoic acid (Z)-, methyl ester & 37.941 & 296 \\
\hline 171 & Methyl stearate & 38.799 & 298 \\
\hline 172 & Heptadecanoic acid, 16-methyl-, methyl ester & 38.799 & 298 \\
\hline 173 & Tridecanoic acid, 12-methyl-, methyl ester & 38.799 & 242 \\
\hline 174 & Methyl tetradecanoate & 38.799 & 242 \\
\hline 175 & Hexadecanoic acid, 15-methyl-, methyl ester & 38.799 & 284 \\
\hline 176 & Pentadecanoic acid, 15-bromo-, methyl ester & 38.799 & 334 \\
\hline 177 & Pentadecanoic acid, methyl ester & 38.799 & 256 \\
\hline 178 & Cyclopentaneundecanoic acid, methyl ester & 38.799 & 268 \\
\hline 179 & Tetradecanoic acid, 12-methyl-, methyl ester & 38.799 & 256 \\
\hline 180 & Octadecanoic acid, 17-methyl-, methyl ester & 38.799 & 312 \\
\hline 181 & Myo-Inositol, 4-C-methyl- & 40.163 & 194 \\
\hline 182 & Myo-Inositol, 2-C-methyl- & 40.163 & 194 \\
\hline 183 & . alpha.-d-6,3-Furanose, methyl-. beta.-d-glucohexodialdo-1,4-fur & 40.163 & 192 \\
\hline 184 & 3-0-Methyl-d-glucose & 40.163 & 194 \\
\hline 185 & D-Epi-Inositol, 4-C-methyl- & 40.163 & 194 \\
\hline 186 & 3-Methylmannoside & 40.163 & 194 \\
\hline 187 & 2-O-Methyl-D-mannopyranosa & 40.163 & 194 \\
\hline 188 & Scyllo-Inositol, 1-C-methyl- & 40.163 & 194 \\
\hline 189 & Methyl 4-0-methyl-d-arabinopyranoside & 40.163 & 178 \\
\hline 190 & Hydroperoxide, 1,4-dioxan-2-yl & 40.163 & 120 \\
\hline 191 & Heptacosane, 1 -chloro- & 41.134 & 414 \\
\hline 192 & Tritetracontane & 41.134 & 604 \\
\hline 193 & 2-methyloctacosane. & 41.134 & 408 \\
\hline 194 & Tetracosane, 11-decyl- & 41.134 & 478 \\
\hline 195 & Tetratetracontane & 41.134 & 618 \\
\hline
\end{tabular}




\begin{tabular}{llll}
\hline 196 & Sulfurous acid, butyl heptadecyl ester & 41.134 & 376 \\
197 & Sulfurous acid, butyl tridecyl ester & 41.134 & 320 \\
198 & Sulfurous acid, butyl tetradecyl ester & 41.134 \\
199 & Sulfurous acid, pentadecylpentyl ester & 41.134 \\
200 & Sulfurous acid, butyl pentadecyl ester & 41.134 & 334 \\
201 & Sulfurous acid, butyl heptadecyl ester & 42.157 & 362 \\
202 & Sulfurous acid, butyl octadecyl ester & 42.157 & 348 \\
203 & Sulfurous acid, butyl hexadecyl ester & 42.157 & 376 \\
204 & Tritetracontane & 42.157 & 390 \\
205 & Heptacosane, 1-chloro- & 42.157 \\
206 & Sulfurous acid, butyl pentadecyl ester & 42.157 \\
207 & Sulfurous acid, octadecylpentyl ester & 42.157 \\
208 & Sulfurous acid, butyl tetradecyl ester & 42.157 \\
209 & Sulfurous acid, hexadecylpentyl ester & 42.157 \\
210 & Sulfurous acid, butyl tridecyl ester & 42.157 \\
\hline
\end{tabular}

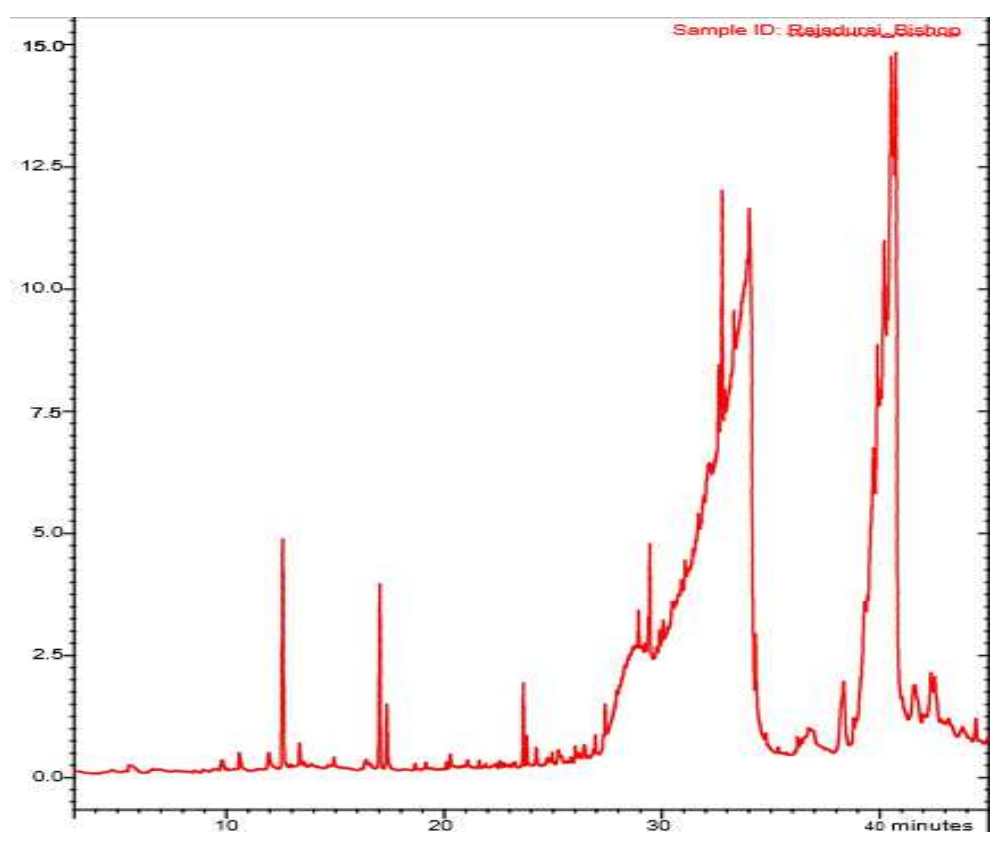

Fig. 1: GCMS analysis of crude extract

\section{CONCLUSION}

This shows that the crude sample may be the mixture of these plant extract. Gas Chromatography and mass spectroscopy analysis put on view the available of various compound with variable molecular weight. This experiment showed that the stronger extraction capacity of methanol could have produced number of bioactive constituents which are plays vital role for many biological activities. This various bioactive compounds might be utilized for the expansion for the drug development which used to treat the kidney stone formation without no side effects, purely in traditional way. At this end it can be concluded that the in vivo studies on the crude extract open up to new ways for natural drug that can be employed for clinical trials which may generate successful results in future.

\section{AUTHORS CONTRIBUTIONS}

All the author have contributed equally

\section{CONFLICTS OF INTERESTS}

All authors have none to declare

\section{REFERENCES}

1. Ayitey Smith E, Addae Mensah IW. Afr J Pharmacol Drug Res 1997;4:7-8.
2. Gill LS. Ethanobotanical uses of plants in Nigeria: university of Benin press: Benin city; 1992. p. 350.

3. Banso A, Adeyemo So. Evaluation of antibacterial properties of tannins isolated from dichrostachys cinerea. Afr J Biotechnol 2007;6:1785-7.

4. Pushpangadan P, Atal CK. Ethano-medico-botanical Investigations ir. Kerala I. Some primitive tribal of Western Ghats and their herbal medicine. J Ethanopharmacol 1984;11:59-77.

5. WHO, IUCN, WWF. Guidelines on the conservation of medicinal plants. Switzerland: IUCN Gland; 1993.

6. Home page on the Internet, World Health Organization. Available from: http://www.who.int/medicines/areas/traditional/definitions/ en/. [Last accessed on 05 Jun 2018]

7. Jana S, shekhawat GS. Phytochemical analysis and antibacterial screening of in vivo and in vitro extracts of Indian medicinal herb: Anethum graveolens. Res J Med Plant 2010;4:206-12.

8. Levey AS, Atkins R, Coresh J. Chronic kidney disease as a global public health problem: approaches and initiatives-a position statement from kidney disease improving global outcomes. Kidney Int Aug 2007;72:247-59. 